\title{
Experimental Research on the Effectiveness of Speed Reduction Markings based on Drivers' Operating Performance: A Driving Simulation Study
}

\author{
Han Ding ${ }^{1, a}$, Li Cui ${ }^{2, b}$, Xiaohua Zhao ${ }^{3, c, *}$, and Wanheng Li $^{1, d}$ \\ ${ }^{1}$ Bridge \& Tunnel Research Center, Research Institute of Highway Ministry of Transport, No. 8 \\ Xitucheng Road, Haidian District, Beijing 100088, P. R. China \\ ${ }^{2}$ Research and Development Center of Transport Industry of Spatial Information Application and \\ Disaster Prevention and Mitigation Technology, CHECC Data Co., Ltd., China Highway \\ Engineering Consultants Corporation, Jiahao Center, No.116, Zizhuyuan Road, Haidian District, \\ Beijing 100097, P. R. China \\ ${ }^{3}$ College of Metropolitan Transportation, Beijing University of Technology, Chaoyang District, \\ Beijing 100124, P.R. China \\ a1143094892@qq.com, bcuili90319@sohu.com, czhaoxiaohua@bjut.edu.cn, dwh.li@rioh.cn
}

Keywords: Traffic Safety, Speed Reduction Markings, Driving Simulation, Decelerating Decision, Operating Performance

\begin{abstract}
Speed reduction markings (SRMs), which are widely used on highways and urban roads in China, are designed to inform drivers of the upcoming road conditions and thus encourage them to reduce travel speed. The objective of this paper is to test the effectiveness of SRMs on drivers' operating performance and decision to decelerate in downhill segments on urban roads. Data of gas and brake pedal use was collected in a driving simulator experiment, and a subjective questionnaire survey was conducted. Two indicators - the operating frequency and operating power-were proposed to evaluate drivers' operating performance due to SRMs. Results of the subjective questionnaire study showed that the majority of subjects were affected by SRMs while driving through downhill segments with distinct roadway grades $(3 \%, 2 \%, 1.5 \%$ and $1 \%$ in experimental scenarios). In terms of the operating frequency, the results of the analysis of variance with repeated measures (rANOVA) and the contrast analysis (S-N-K method) showed that transverse speed reduction markings (TSRMs) were significantly effective in influencing drivers' frequency of letting off the gas pedal when roadway grades of downhill segments were $3 \%, 2 \%$, and $1.5 \%(\mathrm{p}<0.05)$, while longitudinal speed reduction markings (LSRMs) had little effects; both types of SRMs are effective in increasing the frequency of pressing the brake pedal in all four downhill segments. For the operating power, the gas pedal power was significantly affected by TSRMs in all four roadway scenarios; TSRMs also tended to increase the brake pedal power when the roadway grades were $2 \%$ and $1.5 \%$, while both types of SRMs had similar effects in road sections with roadway grades of $3 \%$.
\end{abstract}

\section{Background}

According to (1), as one of the main contributing factors of traffic crashes, speeding alone accounted for 11.7 percent of all traffic fatalities in 2012 in China. To better deal with this issue, speed control devices are usually deployed at sites where speeding-related traffic crashes are more likely to happen. Particularly, speed reduction markings (SRMs) are widely used on highways and urban roads in China, because they are not only capable of alerting drivers to decelerate, but bringing fewer negative effects on drivers and vehicles as well. SRMs are classified into 
longitudinal speed reduction markings (LSRMs) and transverse speed reduction markings (TSRMs), and normally placed within or prior to curves, downhill segments, or other road features where drivers need to control their speed (2,6). Meanwhile, the 2009 U.S. Manual on Uniform Traffic Control Devices (the 2009 MUTCD) also offers detailed illustrations for installation and design principles of SRMs $(3,6)$. Since the design and application of SRMs are unique in China, it is necessary to evaluate the effectiveness of these SRMs in the China National Standard of Traffic Control Devices.

In contrast to the extensive use, it is still unclear whether SRMs could reduce or affect vehicle speeds and to what extent SRMs reduce vehicle speeds. Numerous research studies have been conducted to evaluate the effectiveness of SRMs or the similar pavement lines. For example, Gates et al. (4) researched the effectiveness of transverse bar pavement markings on freeway curves; Katz (5) and Ding et al. (6) evaluated the effects of SRMs by driving simulator experiments. In those research studies, the speed index was selected to observe the effectiveness of SRMs. In actual, the vehicle speed was the most common indicator, which was used to measure the deceleration capability of SRMs in other relevant research $(7,8)$.

However, SRMs belong to nonintrusive speed control devices and are not enforceable in China, which sometimes leads no change in vehicle speeds when drivers are travelling through SRMs. In fact, according to the national standard (2), the main purpose of SRMs is to alert drivers to decelerate. If a driver perceives the existence of SRMs and decides to slow down, one could say that SRMs are effective, regardless of the magnitude of speed change. From this perspective, some researchers turned to probe into the operating principle, and discovered that SRMs would generate some warning or perceptual effects together with speed changes. For example, Kaber et al. (9) pointed out that a driver's conscious control is associated with the driver's reactions to roadway conditions. Zheng (10) stated that SRMs could cause some effects on drivers' perception, as if they were driving faster or the lane became narrower. Those influences on drivers' perception would make them decelerate.

Furthermore, based on the perception-judgment-manipulation mechanism (11), drivers' operating performance is highly associated with perception and judgment. If SRMs make drivers decide to decelerate, drivers will execute related maneuvers. Among all kinds of operating performances, letting off the gas pedal and/or pressing the brake pedal can directly reduce vehicle speed. Consequently, researchers can validate the effectiveness of SRMs on drivers' consciousness and judgment of decelerating by measuring drivers' gas and brake pedal performances. For instance, Ding et al. (12) chose the decelerating operating frequency as one of the indicators to evaluate the effectiveness of SRMs. Other researchers have also discussed the indicators of driving operating. For example, Mulder et al. (13) illustrated drivers' neuromuscular measurements of gas pedal positions and manner by how much force drivers applied to the pedal when driving in the simulator. Rakauskas et al. (14) also used the gas pedal position to measure the effects of cell phone conversations on driving performance.

Existing research has usually been performed through field studies, so that data which reflect vehicle operation status (including speed, acceleration, etc.) could be acquired. Besides, considering the characteristics of nonintrusive speed control devices, the objective of this paper is to test the effectiveness of SRMs on drivers' operating performance and decision to decelerate. Compared to field tests, a simulation study can additionally record drivers' operating performance data, such as accelerating, decelerating, steering, and gear shifting. Field tests could make sure the reliability of speed and operating performance data, however, it is difficult to evaluate the changes in speed or performance due to presence of SRMs because vehicle's 
operating and driver's performance are actually affected by numerous factors, such as presence of SRMs, road alignments, traffic volume and density. The driving simulator study could help us isolate other confounding factors, and observe the effect of SRMs alone on vehicle status or driver's performance. Therefore, a driving simulator experiment was conducted. Particularly, it has to be pointed out that this paper shared the same experiment with (6); nevertheless, this paper used driver's operating data, i.e., accelerator and brake pedals, while the data set of speed and acceleration was analyzed in (6), corresponding to the different research objectives.

\section{Method}

Subjects

In this study, female subjects account for a small number of the total participants, based on the demographic characteristics of licensed drivers in China (15). Twenty-seven male and three female subjects, ages 18 to 42, with an average age of 24.8 years and an average driving experience of 3.3 years, were recruited by advertisement. Specifically speaking, percentages of participants in different age groups $(18-25,25-30$ and $>30)$ were $45 \%, 45 \%$ and $10 \%$, respectively. To capture subjects' actual reactions to the presence of SRMs, participants were not informed of the purpose of the study.

\section{Apparatus}

Technical parameters of a fixed-base driving simulator used in this experiment have been introduced $(6,12)$; the real-time data were collected, including operating performance data (e.g., accelerating, decelerating and steering). The values of gas and brake pedal range from 0 to 1 , denoting the press intensity of these two pedals. For instance, " 0 " denotes that drivers are not pressing, while " 1 " represents that drivers are fully pressing. The data acquisition frequency is $30 \mathrm{~Hz}$, and the virtual scenario was projected onto three large screens, providing a $130^{\circ}$ field of view. Moreover, the driving simulator can also generate various sensory effects to participants, such as visual, auditoria and tactile effects.

\section{Scenarios}

As mentioned in the national standard, SRMs should be placed on or prior to downhill segments. According to the Chinese industrial standard Code for Design of Urban Road Engineering (CJJ37-2012) (16), roadway grades of downhill segments in urban Beijing range from 1\% to 3\%, due to geographic and climatic conditions, and road designers typically choose roadway grades from $1.5 \%$ to $2 \%$. Therefore, this study designed a total of 12 experimental segments [i.e., 3 (No SRMs vs. LSRMs vs. TSRMs) $\times 4$ (roadway grades of $3 \%$ vs. $2 \%$ vs. $1.5 \%$ vs. $1 \%$ )] , and four virtual scenarios were created, as mentioned in (6). Each scenario featured an eight-lane, divided urban expressway, with four one-kilometer tangents and three downhill segments; in all scenarios, the downhill segments were equipped with no SRMs, LSRMs or TSRMs, respectively (see Fig. 1 (a) and (b)). Additionally, roadway grades of three downhill segments in one scenario were the same. Scenarios 1 to 4 were named as 3-PCT (abbreviation for percentage) scenario, 2-PCT scenario, 1.5-PCT scenario and 1-PCT scenario, representing different roadway grades. Detailed designs of two types of SRMs are shown in Fig. 1 (c)-(e), and alignment parameters of virtual scenarios are listed in Table 1. Besides, the vibratory feeling produced by TSRMs could be simulated by the dynamic module in this driving simulator, and subjects would experience such vibratory feelings while traveling through TSRMs. 


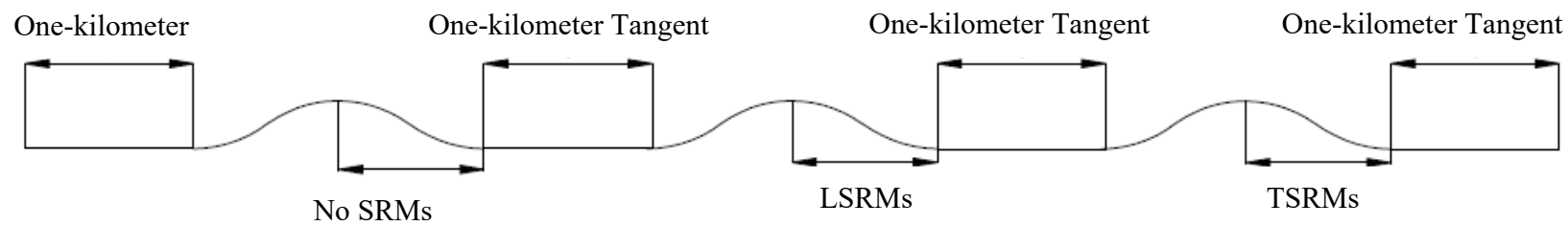

(Downgrade sections)

(a) Profile graph of scenarios

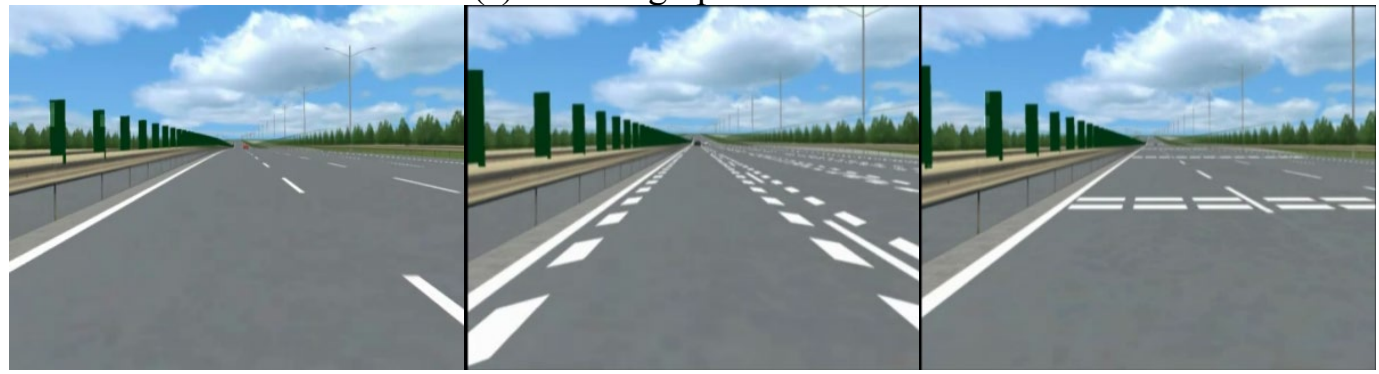

(b) Downhill sections with No SRMs, LSRMs and TSRMs

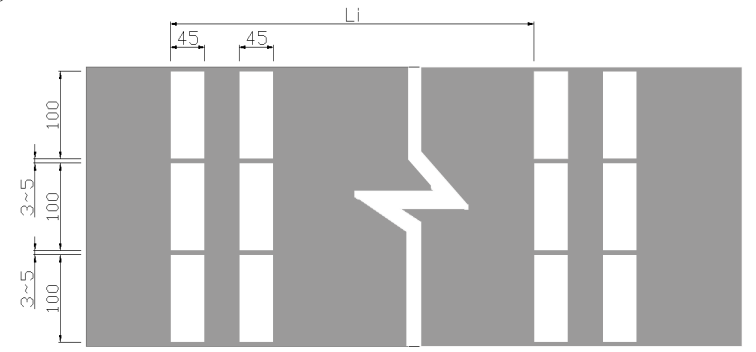

(c) Detailed designs of TSRMs (in cm.)

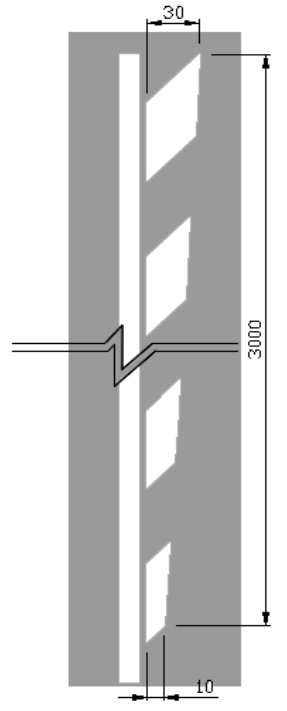

(d) Transition taper of LSRMs (in $\mathrm{cm}$.)

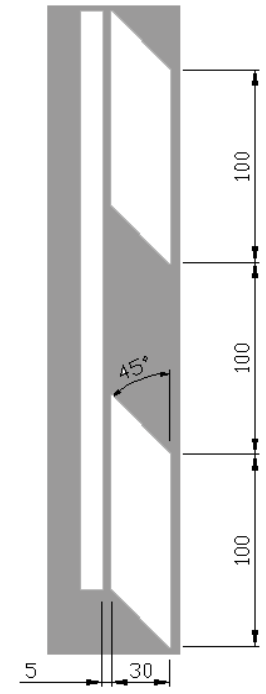

(e) Detailed designs of LSRMs (in cm.)

Fig.1 Scenario design 


\section{Table 1 Alignment parameters of virtual scenarios}

\begin{tabular}{cccccc}
\hline $\begin{array}{c}\text { Scenario } \\
\text { No. }\end{array}$ & $\begin{array}{c}\text { Roadway } \\
\text { grades }(\%)\end{array}$ & $\begin{array}{c}\text { Length of downhill } \\
\text { sections }(\mathrm{m})\end{array}$ & $\begin{array}{c}\text { Elevation of downhill } \\
\text { sections }(\mathrm{m})\end{array}$ & $\begin{array}{c}\text { Length of entire } \\
\text { scenario }(\mathrm{m})\end{array}$ & $\begin{array}{c}\text { Lane } \\
\text { widths }(\mathrm{m})\end{array}$ \\
\hline 1 & 3 & 942.15 & 15 & 9652.90 & \\
2 & 2 & 704.68 & 7 & 8228.08 & 3.75 \\
3 & 1.5 & 659.42 & 5.3 & 7956.52 & \\
4 & 1 & 554.74 & 4.8 & 7328.44 & \\
\hline
\end{tabular}

\section{Procedures}

Since this paper shared the same experiment method with ( 6 ), the procedures have been fully introduced. Subjects were first required to fill out a questionnaire, which recorded their basic information, as well as their physiological and psychological conditions. Then they were required to perform a practice drive for 5-10 minutes on a specific alignment to become familiar with the driving simulator.

After the practice drive, subjects participated in the first driving experiment, in which the operating performance data were collected. During the first experiment, each subject drove from scenario 1 (3-PCT scenario) to scenario 4 (1-PCT scenario) in sequence. Each scenario was driven once, and the entire driving experiment lasted approximately 30 to 40 minutes.

The second driving experiment took place 10 minutes after the first one, so as to avoid fatigue. The main objective of the second experiment was to obtain subjective evaluations of SRMs in downhill segments. Therefore, a subjective questionnaire was designed to ask subjects whether SRMs prompted them to reduce speeds. During the second driving, one operator would sit in the driving simulator. The operator would read questions to subjects and complete the questionnaire when each subject left the road sections equipped with SRMs and entered into following tangents in one scenario, so as to acquire the fresh feeling of subjects about SRMs. It was pre-validated that the tangent was long enough for subjects to recall and answer questions, and subjects could continue their normal driving in following downhill segments. Each scenario would also be driven for once, and the whole driving also lasted for 30 to 40 minute. When the second experiment was finished, subjects left the driving simulator and filled out a questionnaire to report their subjective evaluations of the driving simulator, together with the physiological and psychological status at post-test.

\section{Analysis and Results}

Subjective questionnaires

In this experiment, one questionnaire required subjects to evaluate the performance of the driving simulator subjectively. The evaluation items included the brake, accelerator, scenarios, and SRMs, among other items. The ratings ranged from 0 ("not at all similar to the real world") to 10 ("extremely similar to the real world"). The results are shown in Table 2.

Table 2 Ratings of subjective evaluation of driving simulator

\begin{tabular}{ccccccccc}
\hline & Overall & Steering & Accelerator & Brake & Clutch & Gear & Scenario & SRMs \\
\hline Mean & $\mathbf{7 . 8}$ & $\mathbf{8 . 0}$ & $\mathbf{7 . 7}$ & $\mathbf{7 . 2}$ & $\mathbf{8 . 3}$ & $\mathbf{8 . 3}$ & $\mathbf{8 . 0}$ & $\mathbf{8 . 7}$ \\
\hline
\end{tabular}

The other questionnaire (which was also stated in (6)) was administered to get participants' subjective views of the effectiveness of SRMs, and results are shown in Fig. 2. For example, in the downhill segment with a roadway grade of $3 \%, 83.3 \%$ of subjects were reported to reduce speeds 
due to the presence of LSRMs, and $86.7 \%$ of subjects were influenced by TSRMs. The following was observed:

1) The majority of subjects decelerated for both types of SRMs in all four scenarios.

2) TSRMs in segments with a grade of $3 \%$ were associated with the highest percentage of subjects slowing down, and the percentages of subjects slowing down on a downhill segment with other grades (i.e., $1 \%, 1.5 \%$ and $2 \%$ ) were similar but slightly lower.

3) The effects of LSRMs in all four scenarios were lower than the effects of TSRMs.

4) The percentage of subjects slowing down on a downhill segment with LSRMs apparently decreased as the roadway grades decreased.

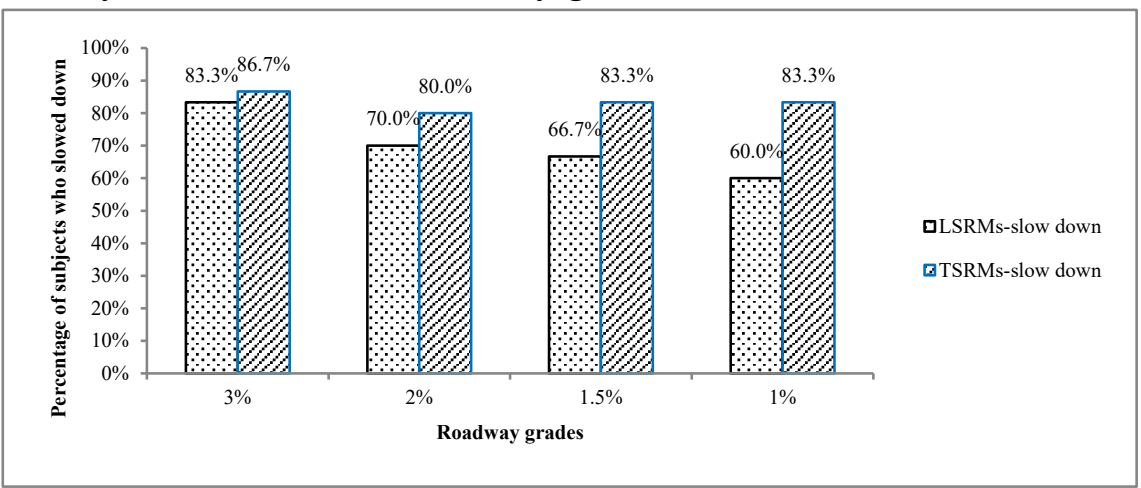

Fig. 2 Percentage of subjects slowing down due to the presence of SRMs

Operating frequency

In this paper, effects of SRMs on drivers' operating performance and driving decisions will be discussed. Among all operating performances, letting off the gas pedal and pressing the brake pedal are two separate actions that can directly control vehicle speeds. Therefore, the operating frequency, measuring how frequently a driver lets off the gas pedal or presses the brake pedal, is analyzed to reflect the change in drivers' decisions.

Frequency of letting off the gas pedal

The results of average frequencies of letting off the gas pedal with respect to different types of SRMs and roadway grades are presented in Fig. 3. It indicates that in the 3-PCT, 2-PCT and 1.5-PCT scenarios, drivers release the gas pedal noticeably less often in road sections paved with TSRMs than sections with No SRMs and LSRMs. In 1-PCT scenario, drivers let off the gas pedal less in road sections with SRMs than those with No SRMs; however, there is no difference between these two types of SRMs. In terms of roadway grades, the average frequency of releasing the gas pedal in the 3-PCT scenario is apparently higher than the ones in the other three scenarios.

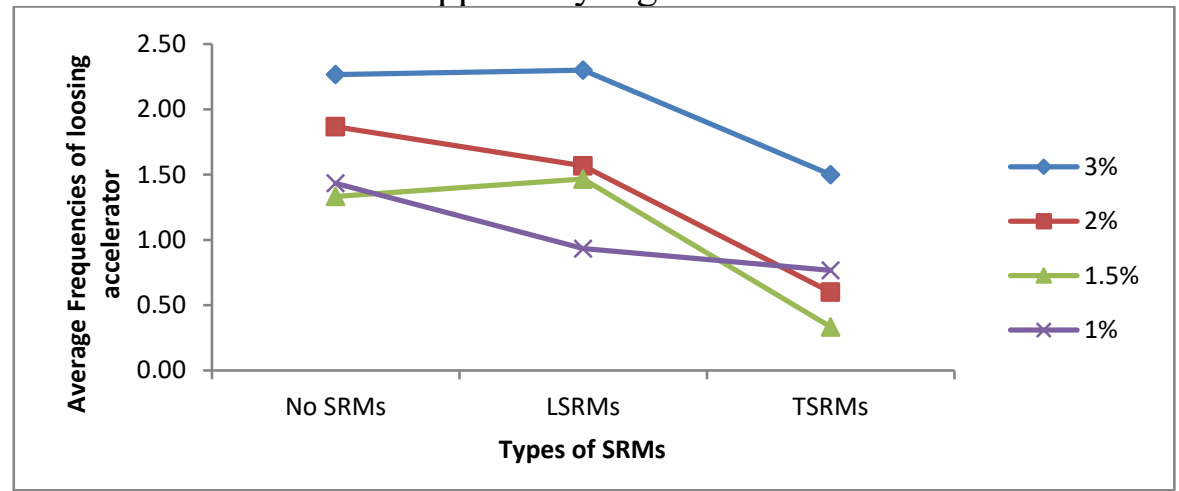

Fig. 3 Average frequencies of letting off the gas pedal 
The analysis of variance with repeated measures (rANOVA) was conducted to compare the impacts of SRMs and roadway grades on average frequencies of letting of the gas pedal. The results revealed significant differences in the indicators among three types of SRMs (No SRMs, LSRMs and TSRMs $)\left(\mathrm{F}_{(2,58)}=26.508 ; P<0.001\right)$ and four roadway grades $(3 \%, 2 \%, 1.5 \%$ and $1 \%)\left(\mathrm{F}_{(3,87)}=11.160 ; P<0.001\right)$.

The ANOVA was further used to identify the differences in the indicators between distinct types of SRMs and roadway grades. In the 3-PCT $\left(\mathrm{F}_{(2,58)}=4.032, P=0.023\right), 2-\mathrm{PCT}\left(\mathrm{F}_{(2,58)}\right.$ $=7.212, P=0.002)$ and 1.5-PCT $\left(\mathrm{F}_{(2,58)}=12.293, P<0.001\right)$ scenarios, results of the ANOVA showed significant main effects of the type of SRMs on the average frequencies. For all three scenarios, a contrast analysis ( $\mathrm{S}-\mathrm{N}-\mathrm{K}$ method) revealed that the average frequencies in the vertical curve with TSRMs were significantly lower than that without SRMs and LSRMs $(\mathrm{P}<0.05$ in all contrasts). The test results suggested that TSRMs had statistically significant effects on the frequency of letting off the gas pedal in downhill segments whose roadway grades were $3 \%, 2 \%$ and $1.5 \%$.

In the 1-PCT scenario, however, results of ANOVA reflected that there was no significant main effect of the type of SRMs on the average frequencies $\left(\mathrm{F}_{(2,58)}=2.932 ; P=0.061\right)$. The test results implied that neither LSRMs nor TSRMs had statistically significant effects on letting off the gas pedal in downhill segments whose roadway grades were $1 \%$.

For LSRMs $\left(\mathrm{F}_{(3,87)}=5.752, P=0.001\right)$ and TSRMs $\left(\mathrm{F}_{(3,87)}=5.868, P=0.001\right)$, analytical results showed significant main effects of roadway grades on the average frequencies. The contrast analysis determined that the average frequencies in the 3-PCT scenario with SRMs are significantly higher than the indicators in the other three scenarios, which inferred that SRMs could induce subjects to let off the gas pedal more in downhill segments with roadway grades of $3 \%$, when compared with the other three roadway grades.

Frequency of pressing the brake pedal

For all drivers, the average frequencies of pressing the brake pedal with respect to different types of SRMs and roadway grades are presented in Fig. 4. There is a clear increasing tendency for this indicator in 3-PCT, 2-PCT and 1.5-PCT scenarios, and drivers press the brake pedal the most in road segments with TSRMs. In the 1-PCT scenario, drivers will press the brake pedal more in road sections with SRMs than without SRMs; however, there is no considerable difference between these two kinds of SRMs.

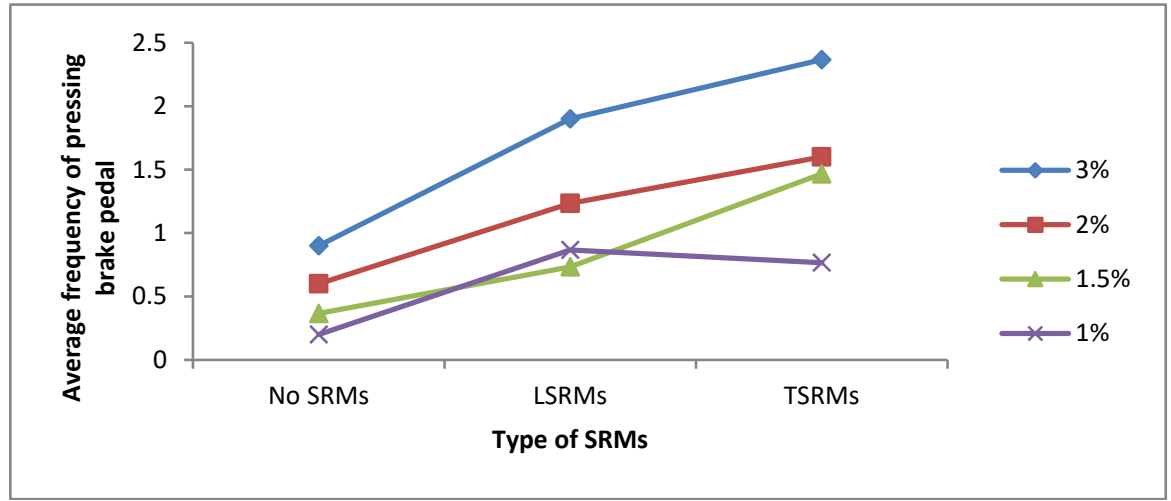

Fig. 4 Average frequencies of pressing the brake pedal

In terms of roadway grades, average frequencies of pressing the brake pedal in the 3-PCT scenario are apparently greater than that in the other three scenarios for both kinds of SRMs; 
particularly, the indicators for TSRMs in the 1.5-PCT and 2-PCT scenarios are higher than the one in the 1-PCT scenario, yet these frequencies are still lower than the frequency related to the 3-PCT scenario.

The rANOVA was used to compare the impacts of SRMs and roadway grades on the average frequency of pressing the brake pedal. The results showed significant differences in the indicator associated with types of SRMs $\left(\mathrm{F}_{(2,58)}=17.265 ; P<0.001\right)$ and roadway grades $\left(\mathrm{F}_{(3,27)}\right.$ $=7.058 ; P=0.001$ ).

The ANOVA was used to evaluate the effectiveness of SRMs with different roadway grades, in terms of average frequencies of pressing the brake pedal. In the 3-PCT $\left(\mathrm{F}_{(2,58)}=7.817\right.$, $P=0.001), 2-\mathrm{PCT}\left(\mathrm{F}_{(2,58)}=9.412, P<0.001\right), 1.5-\mathrm{PCT}\left(\mathrm{F}_{(2,58)}=13.699, P<0.001\right)$ and 1-PCT $\left(\mathrm{F}_{(2,58)}=4.537, P=0.015\right)$ scenarios, results indicated significant main effects of the type of SRMs on the average frequencies. The contrast analysis revealed that the average frequency associated with SRMs was significantly more than the one in the road section without SRMs in 3-PCT, 2-PCT and 1-PCT scenarios, while the average frequency associated with TSRMs was significantly more than the ones in the road segment without SRMs and LSRMs in 1.5-PCT scenario ( $\mathrm{P}<0.05$ in all contrasts). The test results suggested that SRMs had statistically significant effects on pressing the brake pedal in downhill segments whose roadway grades were $3 \%, 2 \%$ and $1 \%$, and only TSRMs had statistically significant effects on pressing the brake pedal in downhill segments whose roadway grades were $1.5 \%$.

In terms of roadway grades, results of the ANOVA showed significant main effects of roadway grades on the average frequencies of braking in road segments equipped with LSRMs $\left(\mathrm{F}_{(3,87)}=7.094, P<0.001\right)$. The contrast analysis determined that the average frequencies of pressing the brake pedal in the 3-PCT scenario with LSRMs are significantly higher than that in the other three scenarios $(\mathrm{P}<0.05$ in all contrasts). The test results inferred that LSRMs could make subjects press the brake pedal more in downhill segments with roadway grades of $3 \%$ than in the three other roadway grades.

For road segments equipped with TSRMs, results of the ANOVA were similar to the results for LSRMs $\left(\mathrm{F}_{(3,87)}=9.291, P<0.001\right)$. The four kinds of roadway grades were divided into three levels: $3 \%, 2 \%$ and $1.5 \%$, and $1 \%$, according to the contrast analysis ( $\mathrm{P}<0.05$ in all contrasts). Specifically, the average frequencies of pressing the brake pedal in the 3-PCT scenario were the highest, while the indicator in the 1-PCT scenario was the lowest. The test results indicate that TSRMs would affect drivers' frequency of pressing the brake pedal in downhill segments with roadway grades of $3 \%$, and such effects would become weaker for roadway grades of $2 \%$ and $1.5 \%$.

\section{Operating power}

As mentioned above, the conclusion was reached that two types of SRMs, either combined or used singly, could affect drivers' operating frequencies in road segments with different roadway grades. Nevertheless, the operating frequency could only describe how many times drivers press gas pedal or brake pedal, and the intensity of pedal pressure and duration of pedal use can also reflect drivers' operating performance, as shown in Fig. 5 (a) and (b). To evaluate the effects of SRMs on drivers' operating performance more comprehensively, the operating power is introduced in this research, which includes the gas pedal power and brake pedal power.

For each subject, the driving simulator records his/her intensity of pressing the gas and brake pedal, and the profile of press intensity can be plotted. For example, Fig. 5 shows press intensity profiles of the brake pedal of one subject in two scenarios. The operating power represents the area 
surrounded by the $\mathrm{x}$-axis, $\mathrm{y}$-axis, and press intensity profile, which is defined simultaneously by press intensity and duration. As the area increases, a driver presses on the gas or brake pedal with more power. Through calculating the area, researchers could compare the effects of SRMs on drivers' operating power.

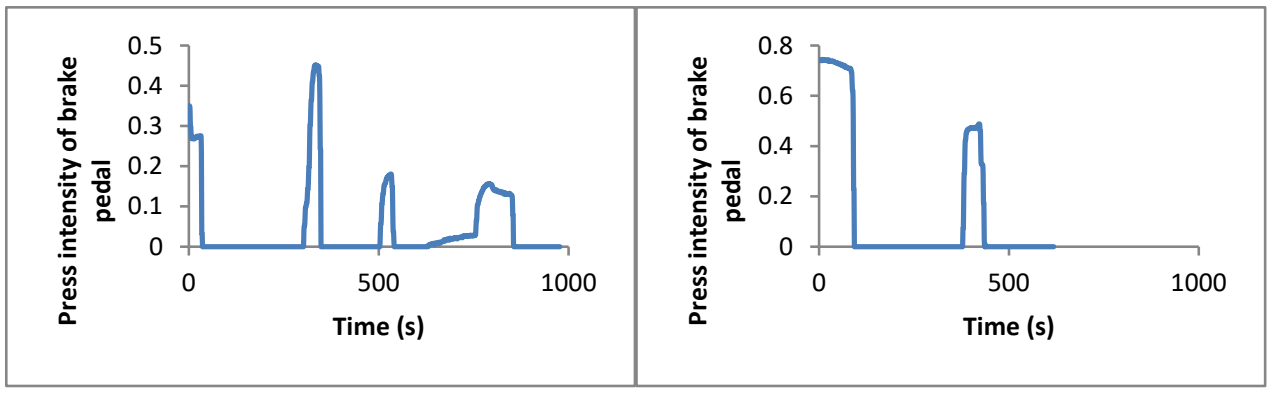

(a)

(b)

Fig. 5 Press intensity of the brake pedal of one subject in two scenarios

\section{Gas pedal power}

The results of the average power of pressing the gas pedal with respect to different types of SRMs and roadway grades are presented in Fig. 6. For all scenarios, SRMs lowered the gas pedal power, which implies that TSRMs have better effects on lowering gas pedal power than LSRMs. Besides, it seems that gas pedal power tends to decline when the roadway grade decreases.

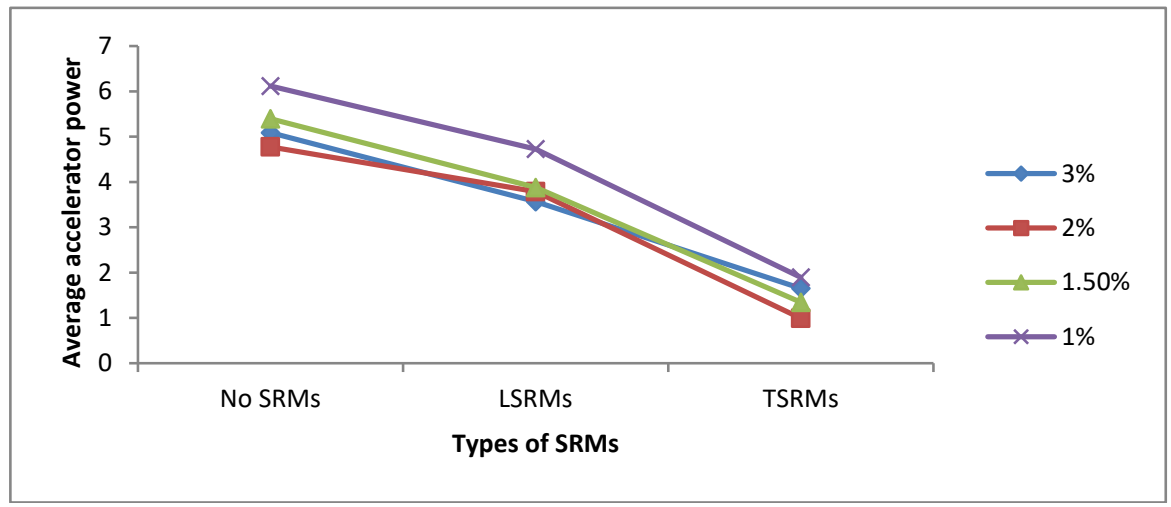

Fig. 6 Average gas pedal powers

The rANOVA was used to compare the impacts of SRMs and roadway grades on gas pedal power. The analytical results showed significant differences in gas pedal power among types of SRMs $\left(\mathrm{F}_{(2,28)}=18.409 ; P<0.001\right)$, while no effects for roadway grades $\left(\mathrm{F}_{(3,27)}=2.613 ; P=0.072\right)$.

For all scenarios, results of ANOVA showed significant main effects of the type of SRMs on gas pedal power $\left(\mathrm{F}_{(2,58)}=10.095, P<0.001\right.$ for the 3-PCT scenario; $\mathrm{F}_{(2,58)}=11.387, P<0.001$ for the 2-PCT scenario; $\mathrm{F}_{(2,58)}=14.454, P<0.001$ for the 1.5-PCT scenario; $\mathrm{F}_{(2,58)}=11.041, P<0.001$ for the 1-PCT scenario). The contrast analysis indicated that the gas pedal power in the downhill segments with TSRMs was significantly lower than that in the other two segments without SRMs and LSRMs $(\mathrm{P}<0.05$ in all contrasts). The test results suggested that TSRMs had statistically significant effects on the drivers' gas pedal operating in downhill segments whose roadway grades were $3 \%, 2 \%, 1.5 \%$ and $1 \%$, while LSRMs did not have such significant effects. 
Brake pedal power

For all drivers, the results of the average power of pressing the brake pedal with respect to different types of SRMs in each scenario are presented in Fig. 7. For 3-PCT, 2-PCT and 1.5-PCT scenarios, SRMs could increase the brake pedal power; specifically, TSRMs might have better effects than LSRMs. Meanwhile, like the results of frequencies of pressing the brake pedal, drivers tend to apply more power in road segments with SRMs than without SRMs in the 1-PCT scenario.

In terms of roadway grades, average brake pedal powers in the 3-PCT scenario are apparently more than that in the other three scenarios for both kinds of SRMs; furthermore, the indicators for TSRMs in the 1.5-PCT and 2-PCT scenarios are higher than the one in the 1-PCT scenario, yet these powers are still lower than the power related to the 3-PCT scenario.

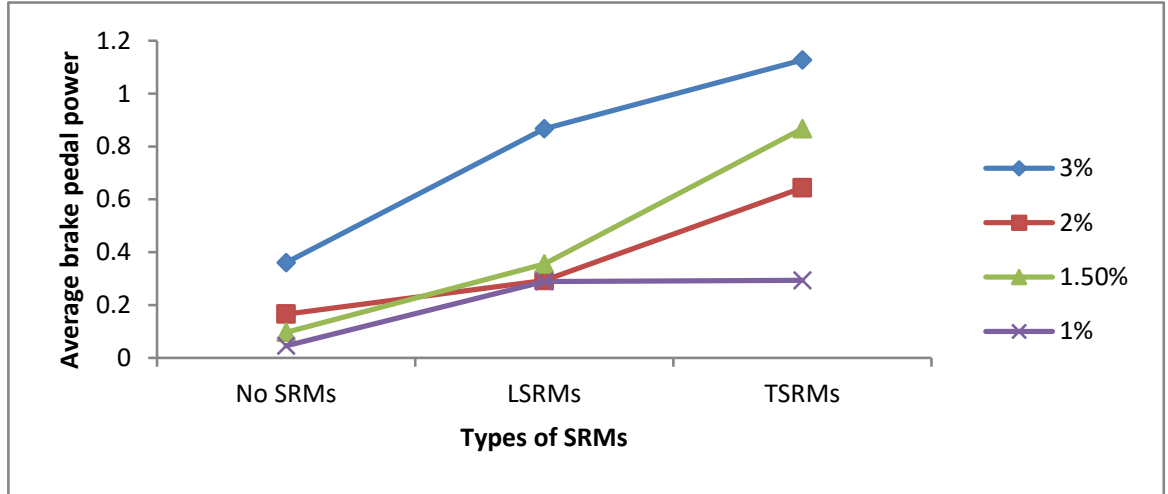

Fig. 7 Average brake pedal power

The rANOVA was used to compare the impacts of SRMs and roadway grades on brake pedal power. The results showed significant differences in brake pedal power among types of downhill segments $\left(\mathrm{F}_{(2,28)}=10.659 ; P<0.001\right)$ and roadway grades $\left(\mathrm{F}_{(3,87)}=15.582 ; P<0.001\right)$.

The ANOVA was used to evaluate the effectiveness of SRMs on brake pedal power in downhill segments with different roadway grades. In the 3-PCT scenario $\left(\mathrm{F}_{(2,58)}=7.140\right.$, $P=0.002$ ), results showed significant main effects of the type of SRMs on brake pedal power. The contrast analysis revealed that the brake pedal power in the vertical curve with SRMs was significantly higher than brake pedal power in the other road segments without SRMs $(\mathrm{P}<0.05$ in all contrasts). The test results suggested that SRMs had statistically significant effects on the brake pedal power in downhill segments whose roadway grades were $3 \%$.

In the 2-PCT $\left(\mathrm{F}_{(2,58)}=11.685, P<0.001\right)$ and 1.5-PCT $\left(\mathrm{F}_{(2,58)}=4.230, P=0.019\right)$ scenarios, the contrast analysis stated that the brake pedal power in the vertical curve with TSRMs was significantly higher than the ones in the other two road segments without SRMs and LSRMs $(\mathrm{P}<0.05$ in all contrasts). The test results stated that TSRMs had statistically significant effects on the brake pedal power in downhill segments whose roadway grades were $2 \%$ and $1.5 \%$.

In the 1-PCT scenario, however, results of the ANOVA reflected that there was no significant main effect of the type of SRMs on the brake pedal power $\left(\mathrm{F}_{(2,58)}=2.785 ; P=0.070\right)$. The test results implied that neither LSRMs nor TSRMs had statistically significant effects on the brake pedal power in downhill segments whose roadway grades were $1 \%$.

In terms of different roadway grades, ANOVA and contrast analysis revealed the same results as average frequencies of pressing the brake pedal. On one hand, $\operatorname{LSRMs}\left(\mathrm{F}_{(3,87)}=7.094\right.$, $P<0.001)$ could make subjects apply more power to the brake pedal in downhill segments with roadway grades of $3 \%$ than in the other three roadway grades $(\mathrm{P}<0.05$ in all contrasts $)$. On the other hand, TSRMs $\left(\mathrm{F}_{(3,87)}=9.291, P<0.001\right)$ would affect drivers' brake pedal power in 
downhill segments with roadway grades of $3 \%$, and such effects would become weaker related to roadway grades of $2 \%$ and $1.5 \%$ as well.

\section{Discussion}

This paper was to evaluate the effectiveness of SRMs on drivers' operating performance and their decisions to decelerate in downhill segments with different roadway grades. The experiment was performed in a driving simulator. In previous works, Xu (17) validated the effectiveness of the Beijing University of Technology driving simulator, and testified that the driving simulator had relative effectiveness on physiological and psychological parameters. Ding et al. (6) had also validated the effectiveness of driving simulator on travel speed in downhill segments equipped with SRMs, compared to the speeds collected in the field. According to Ding et al. (6), the driving simulator had the relative effectiveness in terms of simulating travel speeds, and the speed variation trend in the downhill segments equipped with SRMs was highly similar to the trend in the real road environment.

Two indicators-the operating frequency and operating power-are introduced to measure the effects of SRMs on drivers' operating performance and decision to decelerate. In detail, there is a subtle difference between these two indicators. On one hand, the operating frequency is a more intuitive indicator, which can reflect a driver's consciousness and the decision to decelerate. On the other hand, the operating power is an indirect indicator, which takes intensity and duration into consideration, as well as frequency. Compared with the operating frequency, the operating power on the gas and brake pedals will influence vehicle operations, especially travel speeds.

Considering driver's actions, it is assumed that letting off the gas pedal implies that the driver is conscious of decelerating, while pressing the brake pedal infers that the driver decides to slow down. Referring to results of the operating power, it has been concluded that: 1) TSRMs could significantly decrease the gas pedal power in all kinds of downhill segments; 2) TSRMs could also significantly increase the brake pedal power in 1.5-PCT, 2-PCT and 3-PCT scenarios; and 3) LSRMs could only influence the brake pedal power in 3-PCT scenario. Therefore, it is probable that: 1) TSRMs will influence driver's consciousness of decelerating in all scenarios; 2) TSRMs will also enhance driver's decision to decelerate in all 1.5-PCT, 2-PCT and 3-PCT scenarios; and 3) LSRMs could only strengthen driver's decision to slow down in 3-PCT scenario. In terms of roadway grades, it appeared that driver's consciousness of controlling speed would not be influenced by the roadway grade; however, driver's decision to decelerate will become stronger when the roadway becomes steeper.

It has been mentioned that SRMs have significant effects on the frequency of pressing the brake pedal in the 3-PCT, 2-PCT and 1-PCT scenarios, but only TSRMs have such effects in the 1.5-PCT scenario. However, researchers have hypothesized that both types of SRMs will have effects in the 3-PCT, 2-PCT and 1.5-PCT scenarios, while they are ineffective in the 1-PCT scenario. This inconsistent phenomenon is the original motivation to determine the limitations of the operating frequency and to develop a more comprehensive indicator, representing the operating performances of the gas and brake pedals more precisely. Recognizing that the press intensity and duration can also be affected by SRMs, the concept operating power is finally introduced in this study.

As illustrated above, it has been stated that only TSRMs could reduce the gas pedal power in all road scenarios. Besides, TSRMs can also make drivers apply more power on the brake pedal in downhill segments with roadway grades of $3 \%, 2 \%$ and $1.5 \%$, while LSRMs can have such effects when the roadway grade is 3\%. Therefore, like the results of the previous indicator, LSRMs will have no impacts on drivers' gas pedal operating in all four scenarios, together with brake pedal 
operating in road segments with roadway grades of $2 \%, 1.5 \%$ and $1 \%$. Moreover, LSRMs can influence brake pedal operating only in the 3-PCT scenario. On the contrary, TSRMs could not only lower drivers' gas pedal power in all four scenarios, but increase the brake pedal power when roadway grades are $3 \%, 2 \%$ and $1.5 \%$ as well. It is implied that TSRMs can make drivers execute fewer gas pedal operations and more brake pedal operations in reverse when roadway grades are $3 \%, 2 \%$ and $1.5 \%$; for roadway grades of $1 \%$, TSRMs will simply encourage the driver to perform fewer gas pedal operations, and drivers' attention will not be directed to the brake pedal operating. These assumptions could be tested in future research.

Since the order of the SRMs conditions was the same (no SRM, LSRMs, and TSRMs) in all scenarios, this could lead to a learning effect. Therefore, especially in Fig. 2 (a), one-kilometer tangents, which connected downgrade sections related to different SRMs conditions, were designed to fade subjects' memory or impression of the previous downgrade section, so as to eliminate or reduce the learning effect. In fact, the average and standard deviation of speeds at the beginning of downgrade sections of all subjects were listed in Table 3. It is apparent that scenario, the average and standard deviation of speeds at the beginning of downgrade sections with No SRMs, LSRMs and TSRMs are approximately the same in the same scenario, and it implies that the learning effect is controlled, and it would not substantially influence the results and conclusions. What's more, the analysis of variance with repeated measures (rANOVA) is commonly used to validate the significant effects of treatments in repeated measures. Since the repeated measures were usually organized in the same time sequential order for all subjects, rANOVA was conducted to evaluate the effectiveness of SRMs on drivers' performance.

Table 3 The average and standard deviation of speeds at the beginning of downgrade sections with different SRMs conditions in all scenarios (in $\mathrm{km} / \mathrm{h}$ )

\begin{tabular}{ccccccc}
\hline \multirow{2}{*}{ Roadway grades } & \multicolumn{2}{c}{ No SRMs } & \multicolumn{2}{c}{ LSRMs } & \multicolumn{2}{c}{ TSRMs } \\
& Mean & Std. & Mean & Std. & Mean & Std. \\
\hline $3 \%$ & 104.9 & 12.2 & 104.7 & 14.6 & 108.5 & 13.3 \\
$2 \%$ & 106.5 & 10.6 & 107.9 & 13.5 & 110.7 & 15.4 \\
$1.50 \%$ & 110.2 & 13.7 & 109.1 & 12.6 & 110.1 & 16.8 \\
$1 \%$ & 115.5 & 15.7 & 114.1 & 13.0 & 113.7 & 11.7 \\
\hline
\end{tabular}

The previous research ( $\sigma)$ was repeatedly referenced in this paper, since the methodology was the same. However, the objectives and interest areas were totally different between these two studies. In ( $)$, the impacts of SRMs on vehicle moving status were mainly considered, thus speed and acceleration data were used. Nevertheless, the variation of speed and acceleration was led by driver's consciousness and decision to decelerate, presented as the gas pedal and brake pedal use, and the data and analysis in ( 6 ) was insufficient in mining the relationships between SRMs and driver's operating characteristics. Therefore, the objective of this paper, which was to observe the effects of SRMs on driver's consciousness and decision to decelerate, was confirmed immediately. Additionally, this paper could be viewed as a stretch or inheritance of $(6)$.

Although different data sets were used due to distinct objectives, there were still some similarities in the results of these two studies, and some relationships might be found in the indexes. For example, SRMs were effective when roadway grades were $3 \%, 2 \%$ and $1.5 \%$, and TSRMs had better effects in 2-PCT and 1.5-PCT scenarios, according to ( 6 ). Similarly, TSRMs would affect driver's brake pedal use when roadway grades were $3 \%, 2 \%$, and $1.5 \%$, while LSRMs would only have effects on brake pedal use in 3-PCT scenario. However, the 
similarities between the speed index and driver's gas pedal were not so obvious, compared to the brake pedal power. It was assumed that in this experiment condition, driver's speed behavior was more identical with the brake pedal use, which was also in accordance with the assumptions stated before, that is, pressing the brake pedal infers that the driver decided to slow down; in addition, driver's speed behavior was less uniform with the gas pedal use, since letting off the gas pedal implied that the driver was conscious of decelerating, which might have weaker effects on speed reduction.

\section{Conclusions and Recommendations}

This paper evaluated the effectiveness of SRMs on drivers' operating performance and decisions to decelerate in downhill segments with different roadway grades. By analyzing the data collected in a driving simulator, this research has reached the following conclusions:

- According to the questionnaires, the majority of subjects were encouraged to reduce speeds while driving through downhill segments with LSRMs and TSRMs in all four scenarios. Moreover, TSRMs were more effective than LSRMs in leading subjects to decelerate in all scenarios.

- It is seemed that operating power is an appropriate and comprehensive indicator, which could measure drivers' control performances, since it reflects press frequency, intensity and duration at the same time.

- TSRMs are significantly effective in influencing drivers to let off the gas pedal when roadway grades of downhill segments were $3 \%, 2 \%, 1.5 \%$ and $1 \%$, while LSRMs have no effects. The frequency of pressing the brake pedal was significantly affected by both types of SRMs when the roadway grades of downhill segments were $3 \%, 2 \%$ and $1 \%$, and by TSRMs in road sections with a roadway grade of $1.5 \%$.

- TSRMs can significantly influence drivers' gas pedal operations in all road scenarios, and the brake pedal power when roadway grades are $3 \%, 2 \%$ and $1.5 \%$. LSRMs do not affect drivers' gas pedal operations, and are effective on brake pedal operating only in downhill segments with a roadway grade of $3 \%$.

In summary, this paper discussed the effectiveness of LSRMs and TSRMs on driver's operating performance in downhill segments of urban roads with different roadway grades, based on a driving simulator experiment. In future research, in order to develop guidelines about optimal placement of SRMs the relationship between SRMs and driver's operating performance needs to be evaluated in other road conditions.

\section{Acknowledgements}

This study was funded by the National Key R\&D Program of China (2018YFC0809606).

\section{References}

[1] Statistics of Traffic Crashes in China in 2012. Retrieved Mar 18, 2013, from http://www.tranbbs.com/MResearch/Daten/MResearch_111913.shtml. .

[2] Standardization Administration of the People's Republic of China. Road Traffic Signs and Markings (GB5768-2009). Beijing, 2009.

[3] Federal Highway Administration. Manual on Uniform Traffic Control Devices for Streets and Highways (2009 Edition). U.S. Department of Transportation, 2009

[4] Gates, T. J,X. Qin, and D. A. Noyce. Effectiveness of Experimental Transverse-Bar Pavement 
Marking as Speed-Reduction Treatment on Freeway Curves. In Transportation Research Record: Journal of the Transportation Research Board, No.2056, Transportation Research Board of the National Academies, Washington, D.C., 2008, pp. 95-103. https://doi.org/10.3141/2056-12

[5] Katz, B. J. Peripheral Transverse Pavement Markings for Speed Control. Dissertation submitted to the faculty of the Virginia Polytechnic Institute and State University in partial fulfillment of the requirements for the degree of Doctor of Philosophy in Civil Engineering. Blacksburg, Virginia, 2007.

[6] Ding, H., X. H. Zhao, J. Rong, and J. M. Ma. Experimental Research on the Effectiveness and Adaptability of Speed Reduction Markings in downgrade Sections on Urban Roads: A Driving Simulation Study. Research Report in Transportation Research Center in Beijing University of Technology, 2014. https://doi.org/10.1016/j.aap.2014.11.018

[7] Meyer, E. Application of Optical Speed Bars to Highway Work Zones. In Transportation Research Record: Journal of the Transportation Research Board, No. 1657, Transportation Research Board of the National Academies, Washington, D.C., 1999, pp. 48-54. https://doi.org/10.3141/1657-07

[8] Jiang, J., Z. J. Wang, J. Wu, and J. Lu. Effectiveness and Adaptability Analysis of Typical Speed Control Measures. Journal of Transport Information and Safety, Vol.28, No.155, 2010, pp.96-99.

[9] Kaber, D. B., Y. L. Liang, Y. Zhang, M. L. Rogers, and S. Gangakhedkar. Experimental Driver performance effects of simultaneous visual and cognitive distraction and adaptation behavior. Transportation Research Part F: Traffic Psychology and Behavior, 15 (2012), pp.491-501. https://doi.org/10.1016/j.trf.2012.05.004

[10] Zheng, F. Research on Design and Application of Road Speed Control Facilities. In Master Thesis of Jilin University, Jilin University, Changchun, 2007

[11] Ren, F. T., X. M. Liu, and J. Rong. Traffic Engineering (Second Edition). China Communications Press, Beijing, 2008.

[12] Ding, H. X. H. Zhao, J. Rong, and J. M. Ma. Experimental Research on the Effectiveness of Speed Reduction Markings Based on Driving Simulation: A Case Study. Accident Analysis \& Prevention 60 (2013): 211-218. https://doi.org/10.1016/j.aap.2013.08.007

[13] Mulder, M., M. Mulder, M.M. van Paassen, and D.A. Abbink. Haptic Gas Pedal Feedback. Ergonomics, Vol. 51, No. 11, 2008, pp. 1710-1720. https://doi.org/10.1080/00140130802331583

[14] Rakauskas, M. E., L. J. Gugerty, N. J. Ward. Effects of Naturalistic cell phone conversations on driving performance. Journal of Safety Research 35 (2004): 453-464.

https://doi.org/10.1016/j.jsr.2004.06.003

[15] China Has More Than 0.236 Billion Licensed Drivers and 0.225 Billion Registered Vehicles. Retrieved May 30, 2012, from http://news.mycar168.com/2012/02/263721.html.

[16] The Ministry of Housing and Urban-Rural Development of the People's Republic of China. Code for Design of Urban Road Engineering (CJJ37-2012). Beijing, 2012.

[17] Xu, S. L. The Study on the Discriminating Method of Driving Fatigue Based on Physiological Signal. In Master Thesis of Beijing University of Technology, Beijing University of Technology, Beijing, 2012.

[18] China Has More Than 0.3 Billion Licensed Drivers. Retrieved Nov 27, 2014, from http://www.mps.gov.cn/n16/n1237/n1342/n803715/4287614.html 